\title{
Comparison of Topical Tacrolimus $0.1 \%$ in Pectin Ointment with Clobetasol $0.5 \%$ Ointment in Adults with Moderate to Severe Desquamative Gingivitis: A 4-Week, Randomized, Double-Blind Clinical Trial
}

\author{
Giovanni Corrocher, DDS¹; Gabriele Di Lorenzo, $\mathrm{MD}^{2}$; Pasquale Mansueto, $\mathrm{MD}^{2}$; \\ Nicola Martinelli, $\mathrm{MD}^{3}$; Maria Esposito-Pellitteri, $\mathrm{MD}^{2}$; Stefano Gelio, PharmD ${ }^{4}$; \\ Giorgio Lombardo, $\mathrm{DDS}^{1}$; and Maria Luisa Pacor, $\mathrm{MD}^{3}$ \\ ${ }^{1}$ Dipartimento di Scienze Morfologico-Biomediche, Sezione di Chirurgia Maxillo-Facciale e \\ Odontostomatologia, Università di Verona, Verona, Italy; ${ }^{2}$ Dipartimento di Medicina Clinica e delle \\ Patologie Emergenti, Università di Palermo, Palermo, Italy; ${ }^{3}$ Dipartimento di Medicina Clinica e \\ Sperimentale, Università di Verona, Verona, Italy; and ${ }^{4}$ Servizio Farmacia del Policlinico "G. B. Rossi" di \\ Verona, Verona, Italy
}

\section{ABSTRACT}

Background: Desquamative gingivitis (DG) is a clinical condition characterized by red, painful, glazed, and friable gingiva, which might be a manifestation of some autoimmune mucocutaneous diseases. The time from the development of initial signs of DG to diagnosis can vary from months to years. Based on a literature search, no data concerning patients with DG without signs of autoimmune disease were available.

Objective: The aim of this trial was to compare the efficacy and tolerability of monotherapy with topical tacrolimus $0.1 \%$ in pectin ointment versus clobetasol propionate $0.5 \%$ ointment in adults affected by DG.

Methods: This randomized, double-blind clinical trial was conducted at the Dipartimento di Medicina Clinica e Sperimentale, Università di Verona, Verona, Italy. Patients aged $\geq 18$ years were selected using the department's electronic medical records based on a clinical diagnosis of moderate to severe DG. After a 2 -week washout period, patients were randomly assigned to receive $2 \mathrm{~mL}$ of tacrolimus $0.1 \%$ in pectin (equivalent to $0.2 \mathrm{mg}$ of tacrolimus) or $2 \mathrm{~mL}$ of clobetasol propionate $0.5 \%$ ointment (equivalent to $1 \mathrm{mg}$ of clobetasol) QD for 4 weeks. Evaluations were performed before treatment (baseline), after the treatment period (week 4), and at 2 follow-up visits at weeks 6 and 8. The signs of DG (ie, erythema [atrophy] and desquamation [erosions/ulceration]) were quantified by a blinded investigator using a calculated score based on their surface extension, using a drawing in which the areas of various zones of the mouth were indicated as a percentage of the whole oral mucosa. Severity of erythema and desquamation was rated on a 4 -point scale $(0=$ absent; $1=$ involvement of $<5 \%$ of surface [mild]; $2=5 \%-15 \%$ [moderate]; and $3=$ $>15 \%$ [severe]). The primary end point was the number of patients who achieved remission (severity score of 0 ) in either sign; the secondary end point was the proportions of patients achieving improvement (severity score of 0 or 1 ) in either sign. Before and after treatment, we measured the serum concentrations of tacrolimus and its metabolites with an immunoenzymatic assay kit. Tolerability was assessed using hematology, biochemistry, urinalysis, measurements of systolic/ diastolic blood pressure and heart rate, patient interview, and spontaneous reporting.

Results: A total of 24 patients (18 women, 6 men; all white of Italian origin; age range, 21-65 years; 12 patients per treatment group) were enrolled in the study. In the tacrolimus group, $11(91.7 \%)$ patients achieved remission of erythema and/or desquamation at weeks 4 and 6 ; at week 8 , these rates were $9(75.0 \%)$ and $8(66.7 \%)$, respectively; none of the patients in the clobetasol group achieved remission of either sign at any time point (all, $P<0.001$ ). At weeks 4,6 , and 8 , significantly greater proportions of patients treated

Accepted for publication September 13, 2006.

doi:10.1016/j.clinthera.2006.09.022

0149-2918/06/\$19.00

Printed in the USA. Reproduction in whole or part is not permitted. Copyright (c) 2006 Excerpta Medica, Inc. 


\section{G. Corrocher et al.}

with tacrolimus had improved erythema and desquamation compared with those treated with clobetasol (all, $P<0.001)$. At week 4 , all patients had undetectable serum tacrolimus concentrations $(<1.5 \mu \mathrm{g} / \mathrm{L})$. Six $(50.0 \%)$ patients in the tacrolimus group reported a mild oral burning sensation, and $6(50.0 \%)$ patients in the clobetasol group reported mild mouth dryness. No other adverse events were reported.

Conclusions: The results of this small study suggest that topical tacrolimus $0.1 \%$ in pectin was more effective compared with clobetasol propionate $0.5 \%$ ointment in the treatment of DG. Both treatments were generally well tolerated in the population studied. (Clin Ther. 2006;28:1296-1302) Copyright (C) 2006 Excerpta Medica, Inc.

Key words: tacrolimus, clobetasol, desquamative gingivitis.

\section{INTRODUCTION}

Desquamative gingivitis (DG) is a disorder in which the gingiva are deep red (erythema [atrophy]) and chronic desquamation (erosions/ulcerations) is present. ${ }^{1}$ Desquamative gingivitis is a clinical term, coined by Prinz in $1932,{ }^{2}$ used to describe a chronic, diffuse inflammation of the marginal gingiva, characterized by erosion of interdental papillae and the adjacent gingiva.

The clinical appearance of DG is red, painful, glazed, and friable gingiva, with desquamation and ulceration of the free and attached gingiva. ${ }^{1}$ In 1933 , Meritt ${ }^{3}$ described milder cases of this condition and further characterized the disease process. The term desquamative gingivitis may not always be appropriate because desquamation is not always present, but erosion or even ulceration is. The extent and degree of gingival involvement vary in different cases. In some cases, such as the patients in this study, there can be an intense erythema that predominates over desquamation. In other cases, desquamation is the main feature and the epithelium can be peeled away easily from nonulcerated areas. Patients might be asymptomatic or report mild pain.

Some cases of DG appear to be associated with autoimmune mucocutaneous disease, but lichen planus is the most common (36\%) associated cause. ${ }^{1,4-8}$ There also have been reports of psoriasis, dermatitis herpetiformis, pyostomatitis vegetans, and linear immunoglobulin (Ig) A disease manifesting as DG. ${ }^{9-11} \mathrm{DG}$ might also be a consequence of chemical damage (eg, dental amalgam) or an allergic response. ${ }^{4}$

The time from the development of the signs of DG to a definitive diagnosis can vary from months to years. ${ }^{4}$ DG is of indefinite duration, with periods of remission and exacerbation, and it might heal after several months or the condition might persist for years. ${ }^{1,4-8}$

Both systemic and topical corticosteroids have been used in the treatment of DG. Systemic steroids are the usual choice in cases of extraoral involvement. ${ }^{1}$ However, the oral environment and the consequent difficulty in ensuring adequate, long-term contact of the medicament to the affected sites usually complicate the use of topical treatment. ${ }^{4,6}$

Topical tacrolimus at concentrations of $0.03 \%$, $0.1 \%$, and $0.3 \%$ has been associated with efficacy and tolerability in the treatment of inflammatory skin diseases ${ }^{12-22}$ and has been used for oral diseases associated with autoimmune dermatoses. ${ }^{7,8,23-25}$ Moreover, studies ${ }^{19,20}$ have found that the serum metabolites of tacrolimus were undetectable when the drug was used topically. The drug inhibits the activity of calcineurin, an important intracellular phosphatase and, thereby, T-lymphocyte activation. ${ }^{26,27}$ The results from an openlabel study by Lyon et $\mathrm{al}^{28}$ in 2001 suggested that topical tacrolimus $0.3 \%(\mathrm{n}=11)$ formulated in carmellose sodium paste was efficacious for the management of peristomal pyoderma gangrenosum. This preparation of tacrolimus was described as a more effective and expeditious treatment than topical clobetasol propionate $0.05 \%$ ointment $(\mathrm{n}=13$ ) for peristomal pyoderma gangrenosum. In the patients who responded to topical treatment, the change in mean lesion size from baseline to after treatment was significantly greater in the clobetasol propionate group compared with the tacrolimus treated-group $(P<0.05)$.

Based on a MEDLINE search using the key terms tacrolimus, clobetasol dipropionate, and desquamative gingivitis (years: 1970-2005), no data concerning patients with DG without signs of autoimmune disease were available. The aim of the present 4 -week, randomized, open-label clinical trial was to assess the efficacy and tolerability of monotherapy with topical tacrolimus $0.1 \%$ in pectin compared with clobetasol propionate $0.5 \%$ ointment in adults affected by $\mathrm{DG}$.

\section{PATIENTS AND METHODS}

The protocol of this 4-week, randomized, doubleblind study was approved by the Human Subjects 
Committee of the institutional review board of the Dipartimento di Medicina Clinica e Sperimentale, Università di Verona, Verona, Italy, where the study was conducted. All patients provided written informed consent to participate. The study was conducted in accordance with the principles of the Declaration of Helsinki ${ }^{29}$ and Good Clinical Practice. ${ }^{30}$

\section{Inclusion and Exclusion Criteria}

Patients aged $\geq 18$ years were selected from the database of the Dipartimento di Medicina Clinica e Sperimentale on the basis of a clinical history of moderate to severe $\mathrm{DG}$, defined as blistering gingiva with marked erythema of marginal and attached gingiva, in the absence of other mucosal lesions. In all patients, the diagnosis of DG was a clinical one, using mucosal biopsy performed by the investigators (G.L. and G.C.).

Patients were excluded if they had recurrent vesicles of the skin, blister lesions, or ocular lesions; autoimmune dermatoses with oral mucosa or inflammatory skin or intestinal disease; or the presence of $\mathrm{IgG}$, IgA, or complement factor C3 along the basement membrane on biopsy, indicating gingival lichen planus, pomphoid, mucous membrane, pemphigoid, linear IgA disease, chronic ulcerative stomatitis, and/or plasma cell gingivitis. Patients with any symptom associated with DG (eg, pain) were excluded. Additional exclusion criteria were previous treatment with $\geq 1$ systemic corticosteroid, immunosuppressant, and/or topical glucocorticosteroid and/or tacrolimus within 8 weeks or with phototherapy within 6 weeks before participation in the study. Pregnant or breastfeeding women were also excluded.

\section{Study Design and Medications}

After screening, all patients underwent a 2-week washout period. After run-in, patients were randomly assigned, in a 1:1 ratio using a computer-generated randomization schedule (StatsDirect Ltd., Cheshire, United Kingdom), to receive $2 \mathrm{~mL}$ of tacrolimus $0.1 \%$ in pectin (equivalent to $0.2 \mathrm{mg}$ of tacrolimus) or $2 \mathrm{~mL}$ of clobetasol propionate $0.5 \%$ ointment (equivalent to $1 \mathrm{mg}$ of clobetasol) QD for 4 weeks. Tacrolimus was prepared by a pharmacist (S.G.) at the Servizio Farmacia del Policlinico "G.B. Rossi" di Verona. The content of a tacrolimus capsule $(100 \mathrm{mg})$ was mixed with $100 \mathrm{~g}$ of Luan (Molteni Farmaceutici, Florence, Italy), which contained $1.0 \mathrm{~g}$ of lidocaine chlorhydrate and the following excipients: polyethylene glycol, car- boxymethylcellulose, propylparaoxybenzoate, methylparaoxybenzoate, ethylparaoxybenzoate, and sodium benzoate. Patients were instructed to apply the medications using a latex glove and to refrain from eating or drinking for at least 30 minutes after application of the medications. To maintain blinding, medications were provided in identical packaging.

No rescue medications were allowed throughout the study period. If a patient experienced significant problems, he or she was instructed to visit one of the participating dental surgeons (G.C. or G.L.). Dental surgeons were responsible for ensuring randomization and blinding and for dispensing the medications.

\section{Efficacy Assessments}

All clinical evaluations were performed by a member of the study team (M.L.P.) who was blinded to treatment assignment. Efficacy assessments were performed before (baseline) and after the treatment period (week 4), and twice after the end of treatment (at weeks 6 and 8). Grading of DG was assessed by the blinded evaluator according to the area of the gingiva with erythema and desquamation. The severity of erythema and desquamation were assessed, using a drawing in which the areas of the gingiva were indicated as a percentage of the whole surface of the gingival mucosa, on a 4-point scale $(0=$ absent; $1=$ involvement of $<5 \%$ of the gingival surface [mild]; $2=5 \%-15 \%$ [moderate]; and $3=>15 \%$ [severe]).

\section{Tolerability Assessments}

The tolerability of the study treatments was assessed using hematologic and biochemical testing. Before and after treatment, patients underwent hematologic testing (measurement of hemoglobin concentration and counts of red blood cells, platelets, and white blood cells [Technicon-H1 blood cell counter, Bayer, Leverkusen, Germany]) and biochemical testing (measurement of serum electrolytes [sodium and potassium], kidney function testing [serum creatinine, serum urea, and urinary analysis], liver function testing [serum aspartate aminotransferase, serum alanine transaminase, serum alkaline phosphatase, and serum $\gamma$-glutamyl transpeptidase], and fasting plasma glucose testing), performed with an autoanalyzer (Modular AR, ISE 900 P800, Roche Diagnostics Systems, Basel, Switzerland). Finally, blood concentrations of tacrolimus and its metabolites were measured using a commercial kit (Dade Behring Syva Enzyme Multiplied 


\section{G. Corrocher et al.}

Immunoassay Technique, Liederbach, Germany) and a Cobas Integra 400 analyzer (Roche Diagnostics Systems). The lower limit of detection in the serum was $1.5 \mathrm{ng} / \mathrm{L}$.

Adverse events were also recorded at each visit through spontaneous reports by patients, patient interview (questions were asked about oral burning and oral dryness), and/or direct observation by the investigator (M.L.P.). The investigator rated the severity of the adverse events using a 3 -point scale (mild, moderate, or severe) and the relationship of each adverse event to treatment using the categories unrelated, unlikely, possibly, probably, and almost certainly related to study treatment, based on the investigator's judgment.

\section{Compliance}

Compliance was assessed using evaluation of unused study drug returned after the treatment period.

\section{Statistical Analysis}

Statistical analysis was performed with SPSS version 13.0 (SPSS Inc., Chicago, Illinois). The comparison of quantitative variables, such as age, between the 2 treatment groups was performed by $t$ test. Severity scores for erythema and desquamation expressed the prevalence of severity at each ambulatory evaluation and, because they were considered ordinal scores, were analyzed by nonparametric tests. The proportion of patients who achieved improvement (defined as erythema/ desquamation severity score of 0 or 1 ) during the trial period (baseline, week 4 of treatment, and first and second follow-up evaluations) were analyzed using the Wilcoxon signed rank test. A between-group comparison of the proportions of patients achieving improvement was performed with the Mann-Whitney $U$ test. A value of $P<0.05$ was considered significant.

The primary end point was the number of patients who achieved complete remission (severity score of 0 ) of signs and a difference in prevalence of that outcome of $\geq 75 \%$ between the 2 treatment groups. The secondary end point was the proportions of patients achieving improvement in either sign. A sample size of $24 \mathrm{pa}-$ tients was calculated a priori for a power of $95 \%$, as determined by Altman nomogram. ${ }^{31,32}$

\section{RESULTS}

A total of 24 patients ( 18 women, 6 men; all white of Italian origin; age range, 21-65 years; 12 patients per treatment group) were enrolled in the study. The
2 treatment groups were equally represented for sex (3 [25\%] of 12 in each group were male) and were not statistically different with regard to mean (SD) age (tacrolimus group, 47.3 [14.2] years vs clobetasol group, 54.6 [13.0] years). All enrolled patients completed the study, including the follow-up periods.

The table shows the percentages of patients with each severity score for erythema and desquamation at baseline, after treatment end, and at the first and second follow-up visits. At baseline, all patients in both treatment groups had erythema (tacrolimus group, 3 $[25.0 \%]$ and $9[75.0 \%]$ patients, respectively; clobetasol group, $2[16.7 \%]$ and $10[83.3 \%]$ patients, respectively). However, the proportion of patients with severe desquamation was significantly lower in the clobetasol group compared with that in the tacrolimus group (0 vs $7[58.3 \%] ; P=0.008)$. Compliance was $100 \%$ for each group.

\section{Efficacy}

In the tacrolimus group, $11(91.7 \%)$ patients achieved remission of erythema and/or desquamation at weeks 4 and 6 ; at week 8 , these rates were $9(75.0 \%)$ and $8(66.7 \%)$, respectively. None of the patients in the clobetasol group achieved remission of either sign at any time point (all, $P<0.001$ between groups) (Table).

In the tacrolimus treatment group, at the week-4 evaluation, all patients had improved erythema and desquamation compared with 0 patients at baseline (both signs, absent, 11 [91.7\%]; mild, 1 [8.3\%]; erythema, $P=0.001$; desquamation, $P=0.002$ ) (Table). These improvements were maintained at the 2 followup evaluations (week 6: erythema, absent, 11 [91.7\%]; mild, $1[8.3 \%][P=0.001$ vs baseline $]$; desquamation, absent, $11[91.7 \%]$; mild, 1 [8.3\%] [P=0.002 vs baseline]; week 8: erythema, absent, $9[75.0 \%]$; mild, 3 $[25.0 \%][P=0.002$ vs baseline $]$; desquamation, absent, $8[66.7 \%]$; mild, 4 [33.3\%] [P=0.001 vs baseline]).

In the clobetasol group, the proportion of patients with mild erythema was significantly greater at week 4 compared with baseline (5 $[41.7 \%]$ vs $0 ; P=0.001$ ). This improvement was maintained at the first and second follow-up visits (week 6, mild erythema, 4 [33.3\%]; week 8, $3[25.0 \%] ; P=0.002$ and $P=0.021$, respectively, vs baseline). On the other hand, the proportion of patients with mild or absent desquamation was not increased significantly from baseline at any time point. 


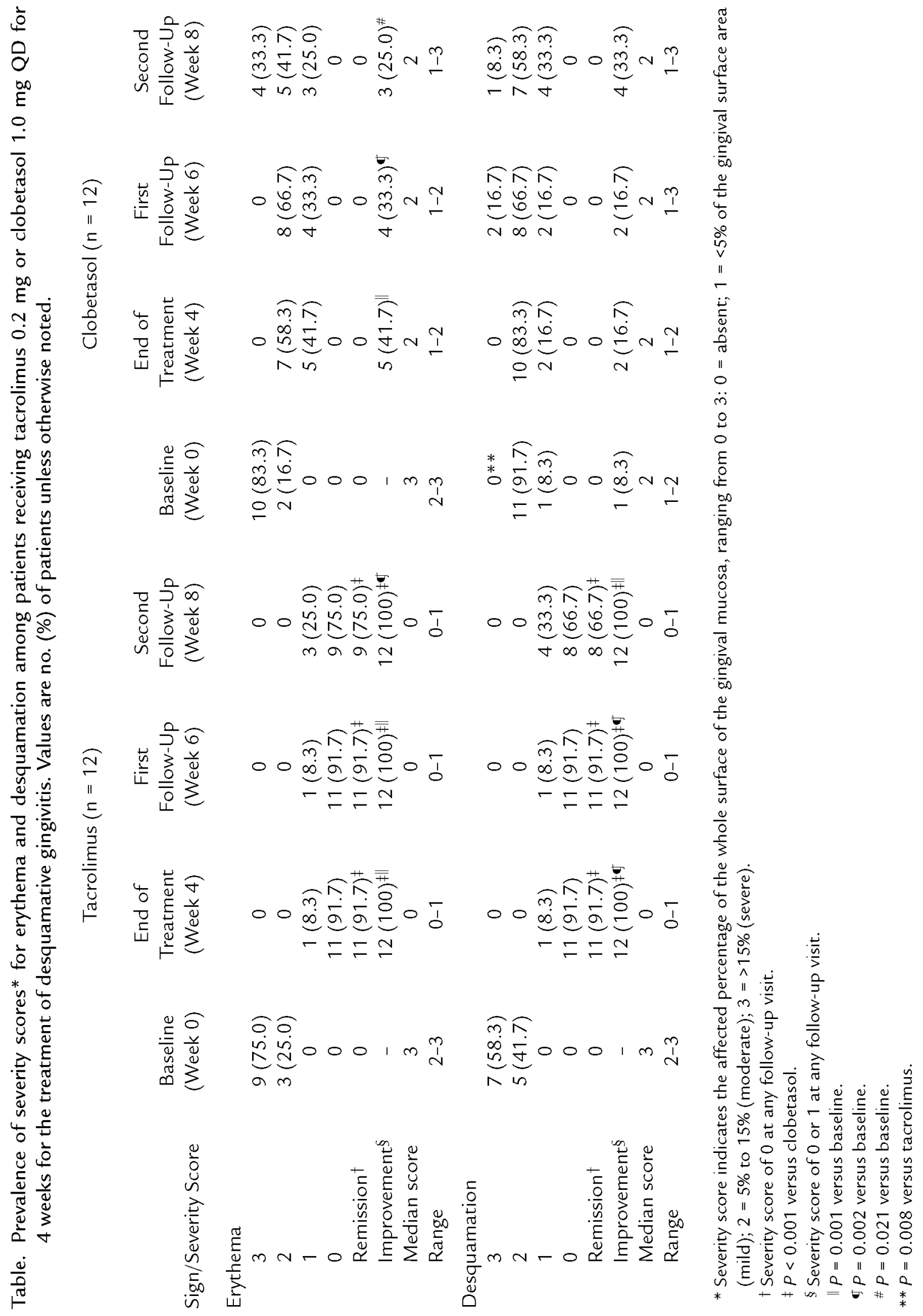




\section{G. Corrocher et al.}

At weeks 4,6 , and 8 , the proportions of patients with improvement in erythema and desquamation were significantly greater in the tacrolimus group compared with the clobetasol group (all, $P<0.001$ ).

\section{Tolerability}

There were no significant changes in hematologic and biochemical indices, blood pressure, or heart rate after 4 weeks of treatment in either group. No severe adverse events were reported in either treatment group. The sensation of oral burning was reported in 6 $(50.0 \%)$ patients treated with tacrolimus, while mouth dryness was reported in $6(50.0 \%)$ patients treated with clobetasol propionate. These adverse events were mild, presented after the initiation of treatment, and resolved as DG improved.

After 4 weeks of treatment, all of the patients treated with tacrolimus had undetectable $(<1.5 \mu \mathrm{g} / \mathrm{L})$ serum concentrations of tacrolimus and its metabolites.

\section{DISCUSSION}

In the present study, topical clobetasol $0.5 \%$ ointment was only partially effective in controlling erythema and desquamation of DG, in contrast to the results of other studies. ${ }^{33,34}$ There have been reports of relapse of the oral lesions after cessation of topical tacrolimus treatment in patients affected by oral lichen planus (in $8[100 \%]$ patients after 12 months, ${ }^{8}$ in $4[36 \%]$ patients within $1-2$ weeks, ${ }^{35}$ and in 5 [83\%] patients within 3-8 weeks $^{36}$ ). The concentration of tacrolimus used in those studies were $0.1 \%$, 36 and $0.03 \%, 0.1 \%$, and $0.3 \%{ }^{35}$ In our study, after 6 weeks of follow-up, a relapse of DG was not observed in either treatment group. However, the chronic nature of the autoimmune mucocutaneous diseases that are often associated with DG might require long-term (6-12 weeks) use of topical tacrolimus, which could be associated with immunosuppressant toxicity, including malignancy.7,8,12-28,35,36 The specific cause (ie, oral lichen planus, pemphigoid) of DG was not determined in the 24 patients in this study.

The principal limitations of this study were the small number of patients treated and a duration of treatment possibly too brief to identify possible adverse events due to long-term use of the drug. Another limitation was the lack of patients' evaluation of effectiveness. It may have also been better to compare topical tacrolimus in pectin against clobetasol in pectin. However, we were unable to find clobetasol in this formulation. Additional randomized, doubleblind, controlled studies, especially comparative and long-term studies, are needed to test this tacrolimus preparation in the treatment of $D G$.

\section{CONCLUSIONS}

The results of this small, randomized, double-blind clinical trial suggest that topical tacrolimus $0.1 \%$ in pectin was more effective compared with clobetasol propionate $0.5 \%$ ointment in the treatment of $\mathrm{DG}$. Both treatments were generally well tolerated in the population studied.

\section{ACKNOWLEDGMENTS}

This study was supported by grants from the Ministero Italiano University of Ricerca, Rome, Italy, to Drs. Pacor and Di Lorenzo. Funding was used to purchase study drugs and software. Clobetasol propionate $0.5 \%$ ointment was purchased from GlaxoSmithKline, Verona, Italy.

\section{REFERENCES}

1. Robinson NA, Wray D. Desquamative gingivitis: A sign of mucocutaneous disorders-a review. Aust Dent J. 2003;48: 206-211.

2. Prinz H. Chronic diffuse desquamative gingivitis. Dentol Cosmos. 1932;74:331-333.

3. Meritt $\mathrm{AH}$. Chronic desquamative gingivitis. J Periodontol. 1933;4:30-34.

4. Vaillant L, Chauchaix-Barthes S, Huttenberger B, et al. Chronic desquamative gingivitis syndrome: Retrospective analysis of 33 cases [in French]. Ann Dermatol Venereol. 2000;127:381-387.

5. Scully C, Porter SR. The clinical spectrum of desquamative gingivitis. Semin Cutan Med Surg. 1997;16:308-313.

6. Chandra RV, Pandurang P, Bhat KM. Labial veneers in the management of desquamative gingivitis: Report of a case. J Contemp Dent Pract. 2004;5:122-132.

7. Lener EV, Brieva J, Schachter M, et al. Successful treatment of erosive lichen planus with topical tacrolimus. Arch Dermatol. 2001;137:419-422.

8. Olivier V, Lacour JP, Mousnier A, et al. Treatment of chronic erosive oral lichen planus with low concentrations of topical tacrolimus: An open prospective study. Arch Dermatol. 2002;138:1335-1338.

9. Jones LE, Dolby AE. Desquamative gingivitis associated with psoriasis. J Periodontol. 1972;43:35-37.

10. Wray D. Pyostomatitis vegetans. Br Dent J. 1984;157:316318.

11. Porter SR, Scully C, Midda M, Eveson JW. Adult linear immunoglobulin A disease manifesting a desquamative gin- 


\section{Clinical Therapeutics}

givitis. Oral Surg Oral Med Oral Pathol. 1990;70:450-453.

12. Reich K, Vente C, Neumann C. Topical tacrolimus for pyoderma gangrenosum. Br J Dermatol. 1998; 139:755-757.

13. Schuppe HC, Homey B, Assmann T, et al. Topical tacrolimus for pyoderma gangrenosum. Lancet. 1998;351: 832.

14. Ruzicka T, Assmann T, Homey B. Tacrolimus: The drug for the turn of the millennium? Arch Dermatol. 1999; 135:574-580.

15. Richter-Hintz D, Schuppe HC, Homey B, et al. Topical tacrolimus (FK 506) is effective in the treatment of pyoderma gangrenosum. J Am Acad Dermatol. 2000;42:304-305.

16. Goldman D. Tacrolimus ointment for the treatment of steroid-induced rosacea: A preliminary report. J Am Acad Dermatol. 2001;44:995-998.

17. Yamamoto T, Nishioka K. Topical tacrolimus: An effective therapy for facial psoriasis. EurJ Dermatol. 2003; 13:471-473.

18. Bamford JT, Elliott BA, Haller IV. Tacrolimus effect on rosacea. J Am Acad Dermatol. 2004;50:107-108.

19. Anderson BE, Marks JG Jr, Mauger DT. Efficacy of tacrolimus ointment in the prevention and treatment of contact dermatitis. Dermatitis. 2004; 15:158-159.

20. Pacor ML, Di Lorenzo G, Martinelli $\mathrm{N}$, et al. Comparing tacrolimus ointment and oral cyclosporine in adult patients affected by atopic dermatitis: A randomized study. Clin Exp Allergy. 2004;34:639-645.

21. Schachner LA, Lamerson C, Sheehan MP, et al, for the US Tacrolimus Ointment Study Group. Tacrolimus ointment $0.03 \%$ is safe and effective for the treatment of mild to moderate atopic dermatitis in pediatric patients: Results from a randomized, doubleblind, vehicle-controlled study. Pediatrics. 2005;116:e334-e342. Available at: http://pediatrics.aapublications.org/ cgi/content/full/116/3/e334. Accessed September 2005.
22. Beck LA. The efficacy and safety of tacrolimus ointment: A clinical review. J Am Acad Dermatol. 2005;53 (Suppl 2):S165-S170.

23. Nazzaro G, Cestari R. Topical tacrolimus ointment in ulcerative lichen planus: An alternative therapeutic approach. Eur J Dermatol. 2002;12:321.

24. Morrison L, Kratochvil FJ III, Gorman A. An open trial of topical tacrolimus for erosive oral lichen planus. J Am Acad Dermatol. 2002; 47:617-620.

25. Hodgson TA, Sahni N, Kaliakatsou $F$, et al. Long-term efficacy and safety of topical tacrolimus in the management of ulcerative/erosive oral lichen planus. Eur J Dermatol. 2003; 13:466-470.

26. Tocci MJ, Matkovich DA, Collier KA, et al. The immunosuppressant FK506 selectively inhibits expression of early $T$ cell activation genes. I Immunol. 1989;143:718-726.

27. Panhans-Gross A, Novak N, Kraft S, Bieber T. Human epidermal Langerhans' cells are targets for the immunosuppressive macrolide tacrolimus (FK506). J Allergy Clin Immunol. 2001; 107:345-352.

28. Lyon CC, Stapleton M, Smith AJ, et al. Topical tacrolimus in the management of peristomal pyoderma gangrenosum. J Dermatol Treat. 2001; 12:13-17.

29. World Medical Association Declaration of Helsinki: Recommendations Guiding Medical Doctors in Biomedical Research Involving Human Subjects [WMA Web site]. FerneyVoltaire, France: WMA; 1989. Available at: http://www.wma.net/e/ policy/b3.htm. Accessed October 2004.

30. European Agency for the Evaluation of Medicinal Products, International Conference on Harmonisation-World Health Organization. Guideline for Good Clinical Practice [EMEA Web site]. ICH Topic E6. Geneva, Switzerland: $\mathrm{WHO} ; 2002$. Available at: http://www.emea.eu.int. Accessed July 1996.

31. Armitage P, Berry G, Matthews JN. Statistical Methods in Medical Research. 4th ed. Oxford, UK: Blackwell Science; 2002.

32. Altman DG. Statistics and ethics in medical research: III. How large a sample? BMJ. 1980;281:1336-1338.

33. Lo Muzio L, della Valle A, Mignogna $M D$, et al. The treatment of oral aphthous ulceration or erosive lichen planus with topical clobetasol propionate in three preparations: A clinical and pilot study on 54 patients. J Oral Pathol Med. 2001;30: 611-617.

34. Gonzalez-Moles MA, Ruiz-Avila I, Rodriguez-Archilla $A$, et al. Treatment of severe erosive gingival lesions by topical application of clobetasol propionate in custom trays. Oral Surg Oral Med Oral Pathol Oral Radiol Endod. 2003;95:688-692.

35. Rozycki TW, Rogers RS III, Pittelkow $M R$, et al. Topical tacrolimus in the treatment of symptomatic oral lichen planus: A series of 13 patients. J Am Acad Dermatol. 2002;46:27-34.

36. Vente C, Reich K, Rupprecht R, Neumann C. Erosive mucosal lichen planus: Response to topical treatment with tacrolimus. BrJ Dermatol. 1999;140:338-342.

Address correspondence to: Gabriele Di Lorenzo, MD, Dipartimento di Medicina Clinica e delle Patologie Emergenti, Università di Palermo, Via del Vespro, 141-90127 Palermo, Italy. E-mail: dilo601@unipa.it 